\title{
Dense Motion Analysis in Fluid Imagery
}

\author{
T. Corpetti, É. Mémin, and P. Pérez \\ 1 IRISA/Université de Rennes I, Campus Universitaire de Beaulieu 35042 Rennes \\ Cedex, France \\ \{tcorpett,memin\}@irisa.fr \\ 2 Microsoft Research Center, 7 J J Thomson Avenue, \\ Cambridge CB3 0FB, UK \\ pperez@microsoft.com
}

\begin{abstract}
Analyzing fluid motion is essential in number of domains and can rarely be handled using generic computer vision techniques. In this particular application context, we address two distinct problems. First we describe a dedicated dense motion estimator. The approach relies on constraints issuing from fluid motion properties and allows us to recover dense motion fields of good quality. Secondly, we address the problem of analyzing such velocity fields. We present a kind of motionbased segmentation relying on an analytic representation of the motion field that permits to extract important quantities such as singularities, stream-functions or velocity potentials. The proposed method has the advantage to be robust, simple, and fast.
\end{abstract}

\section{Introduction}

Since several years, the analysis of video sequences showing the evolution of fluid phenomenon gave rise to a great attention from the computer vision community [4|11|15 23|24]. The applications concern domains such as experimental visualization in fluid mechanics, environmental sciences (oceanography, meteorology, ...), or medical imagery.

In all these application domains, it is of primary interest to measure the instantaneous velocity of fluid particles. In oceanography one is interested to track sea streams and to observe the drift of some passive entities [9]. In meteorology, both operational and experimental, the task under consideration is the reconstruction of wind fields from the displacements of clouds as observed in various satellite images [19]. In medical imaging the issue is to visualize and analyze blood flow inside the heart, or inside blood vessels [21. The images involved in each domain have their own characteristics and are provided by very different sensors. The huge amount of data of different kinds available, the range of applicative domains involved, and the technical difficulties in the processing of all these peculiar image sequences explain the interest of researchers of the image analysis community.

Extracting dense velocity fields from fluid images can rarely be done with the standard computer vision tools, which are originally designed for quasi-rigid 
motions with stable salient features. These generic approaches are based on a brightness constancy assumption and a spatial smoothness of the motion field. In contrast, fluid images usually exhibit high spatial and temporal distortions of luminance patterns. The design of alternate approaches dedicated to fluid motion thus constitutes a widely open domain of research. The first part of this paper is a contribution in that direction.

Once given a reliable description of the fluid motion, an other problem of interest consists in the extraction and the characterization of the critical - or singular - points of the flow. These points are the centers of kinematical events such as swirl, vortices, or sinks/sources. The knowledge of all these points is precious to understand and predict the flows of interest, but it also provides compact and hierarchical representations of the flow [11. We propose, in the second part of this paper, a method to obtain such characteristics from a dense velocity field. This method is based on an analytic representation of the motion field, using the Rankine model.

This paper is organized as follows. In Section 2, we describe the dedicated technique to estimate a dense motion field for fluid phenomenon. The aim of Section 3 is first to present some properties of $2 D$ motion fields, and then to show how quantities such as singularities, stream-function or velocity potential can be extracted analytically. Then, Section 4 presents a method to extract singular points and their associated parameters from dense motion fields. In section 5, we finally present some experimental results on meteorological sequences.

\section{Fluid Optical Flow Estimator}

Optical flow estimation aims at recovering the apparent displacement field between two consecutive frames in an image sequence. Let $\boldsymbol{w}$ denote the unknown displacement field defined over the continuous plane domain $s \in \Omega$ and $f(s, t)$ the luminance function at point $s$ assumed to be continuous in space and time. The most accurate optical flow estimators used in computer vision are issued from Horn and Schunck method [13. They are defined as the minimizer of an energy function $H=H_{1}+H_{2}$ composed of two terms. The first one assumes the constancy of the luminance of a point along its trajectory $\left(\frac{d f}{d t}=0\right)$. This so-called optical flow constraint (OFC) is captured by letting:

$$
H_{1}(\boldsymbol{w}, f)=\int_{\Omega} \rho\left[\boldsymbol{\nabla} f(\boldsymbol{s}) \cdot \boldsymbol{w}(s)+\frac{\partial f(\boldsymbol{s})}{\partial t}\right] d \boldsymbol{s} .
$$

The penalty function $\rho$ is usually the $L_{2}$ norm but it may be changed to a robust function attenuating the effect of data that deviate significantly from the ofCbased data-model [517]. The second term $\mathrm{H}_{2}$ of the energy function is usually a standard first-order spatial smoothness term:

$$
H_{2}(\boldsymbol{w})=\alpha \int_{\Omega} \rho(\|\boldsymbol{\nabla} \boldsymbol{w}\|) d \boldsymbol{s},
$$

where $\alpha>0$ is a parameter controlling the balance between the smoothness and the global adequacy to the brightness constancy assumption. Function $\rho$ may be 
the quadratic penalty if the searched solution is smooth everywhere or a robust norm function if one wants to handle implicitly the spatial discontinuities of the field 514 16.

The standard dense estimator defined as the minimizer of $H$ is generic. It is only based on the assumption of luminance conservation and of first-order spatial smoothness of the motion. Our aim is to devise a similar estimator that captures some specificities of image sequences with fluid motion.

\subsection{Continuity Equation}

As mentioned above, local deviations from the data-model as those occurring in small occlusion areas, can be handled with a robust cost function. In fluid imagery, the problem is much more complex. Image sequences representing fluid phenomena exhibit areas where the luminance function undergoes high temporal variations along the motion. These areas are often the center of tridimensional motions that cause the appearance or the disappearance of fluid matter within the bidimensional visualization plane. These regions are associated to divergent motions which influence greatly the shape of the velocity field in large surrounding areas. An accurate estimation of the 2D apparent motion in such regions is therefore of the highest importance and is hardly possible with the optical-flow constraint.

Instead of sticking to the intensity conservation assumption, we propose here to rely on the fluid law of mass conservation, also known as continuity equation:

$$
\frac{\partial \chi}{\partial t}+\operatorname{div}(\chi \mathbf{v})=0
$$

where $\chi$ denotes the density of the fluid, $\mathbf{v}$ its $3 \mathrm{D}$ velocity and $\operatorname{div} \mathbf{v}=\frac{\partial u}{\partial x}+\frac{\partial v}{\partial y}+\frac{\partial w}{\partial z}$ stands for the divergence of the vector field $\mathbf{v}=(u, v, w)$. Simple manipulations yield the alternative rewriting:

$$
\frac{d \chi}{d t}+\chi \operatorname{div} \mathbf{v}=0
$$

When the divergence of the 3D apparent flow vanishes, this equation is of the same form as the $2 \mathrm{D}$ optical flow constraint on luminance. The continuity equation originally introduced in 20] as a data model for motion estimation of intensity time varying images has been since incorporated in several works. It has been considered in the context of fluid imagery either for satellite meteorological images 47/24] or for experimental fluid mechanics [23]. It has also been introduced in medical imaging domain to recover $3 \mathrm{D}$ deformation fields of the heart [21] or to analyze blood flow [1]. In all these cases, this model has been proved appealing an alternative to standard luminance constancy assumption.

The use of continuity equation for image sequences analysis relies on two hypotheses. First, the luminance function is assumed to be directly related to a passive quantity transported by the fluid. Secondly, the continuity equation which holds in 3D, is assumed to hold as well for the bidimensional motion field 
captured by the image sequence. This latter assumption has been theoretically established in the case of transmittance imaging by Fitzpatrick 10] and extended by Wildes et al in 23. The first assumption is difficult to validate, especially in meteorological images due to the complexity and the heterogeneity of the underlying physical processes. Nevertheless, as shown by several works, the use of the continuity equation in the case of meteorological data is appealing 424 . As the brightness consistancy is obviously not verified in that type of images, the equation of continuity provides us with an interesting alternative data-model. Instead of expressing a point-wise conservation of the luminance along the motion, this alternative model assumes the conservation of the total luminance of any moving elements of the image. This constraint reads:

$$
\frac{d f}{d t}+f \operatorname{div} \boldsymbol{w}=0
$$

However, as OFC-based data models, a data-model based on the continuity equation is highly sensitive to the presence of noise and is very likely not to hold everywhere. Also, due to its differential nature, the continuity equation is not valid in case of large displacements. In fact, this equation concerns velocity and not displacement [18. Unlike the brightness constancy expressed as $f(\boldsymbol{s}+\boldsymbol{d}(s), t+$ $\Delta t)-f(s, t)=0$, which is explicitly based on displacement, the continuity equation (5), as it stands, cannot serve as the basis of an incremental datamodel embedded in hierarchical estimation schemes. To cope with this problem, let us assume that the velocities are constant between the instants $t$ and $t+\Delta t$. In that case, equation (5) constitutes a simple first-order differential equation which can be integrated from time $t$ to time $t+\Delta t$ along trajectories [7]. Setting $\Delta t=1$ for notational convenience, and incorporating the integral constraint thus obtained in a robust penalty function yields a new data-term:

$$
H_{1}(\boldsymbol{w})=\int_{\Omega} \rho\{f(\boldsymbol{s}+\boldsymbol{w}(\boldsymbol{s}), t+1) \exp (\operatorname{div} \boldsymbol{w}(\boldsymbol{s}))-f(\boldsymbol{s}, t)\} d \boldsymbol{s} .
$$

We now turn to the definition of the smoothness prior to be used in conjunction with this new data energy term.

\subsection{Adapted Div-Curl Regularization}

By using Euler-Lagrange conditions of optimality, it is readily demonstrated that the standard first-order regularization functional $\alpha \int_{\Omega}\|\boldsymbol{\nabla} \boldsymbol{w}(\boldsymbol{s})\|^{2} d \boldsymbol{s}$ is equivalent from the minimization point of view, to the so-called div-curl regularization functional 22]:

$$
\alpha \int_{\Omega}\left([\operatorname{div} \boldsymbol{w}(s)]^{2}+[\operatorname{curl} \boldsymbol{w}(s)]^{2}\right) d s,
$$

where $\operatorname{div} \boldsymbol{w}=\frac{\partial u}{\partial x}+\frac{\partial v}{\partial y}$ and $\operatorname{curl} \boldsymbol{w}=\frac{\partial v}{\partial x}-\frac{\partial u}{\partial y}$ are respectively the divergence and the vorticity of the vector field $\boldsymbol{w}=(u, v)$. 
A first-order regularization therefore penalizes the amplitude of both the divergence and the vorticity of the vector field. For fluid motion estimation, this does not seem appropriate since the apparent velocity field usually exhibits "concentrations" of vorticity and/or divergence. In addition, an under-estimation of the divergence would be all the more problematic in our case, because the data model includes an explicit use of this quantity. For these reasons, it would seem more appropriate to rely on second-order div-curl regularization 1222 :

$$
\int_{\Omega}\left(\|\boldsymbol{\nabla} \operatorname{div} \boldsymbol{w}(s)\|^{2}+\|\nabla \operatorname{curl} \boldsymbol{w}(s)\|^{2}\right) d s
$$

This regularization is nevertheless more difficult to implement. As a matter of fact, associated Euler-Lagrange equation is composed with two fourth-order coupled PDE's, which are tricky to solve numerically. We propose to simplify the problem by introducing auxiliary functions, and defining the alternative functional:

$$
H_{2}(\boldsymbol{w}, \xi, \zeta)=\alpha \int_{\Omega}|\operatorname{div} \boldsymbol{w}-\xi|^{2}+\lambda \rho(\|\nabla \xi\|)+\alpha \int_{\Omega}|\operatorname{curl} \boldsymbol{w}-\zeta|^{2}+\lambda \rho(\|\nabla \zeta\|) .
$$

The new auxiliary scalar functions $\xi$ and $\zeta$ can be respectively seen as estimates of the divergence and the curl of the unknown motion field, and $\lambda$ is a positive parameter. The first part of each integral encourages the displacement to comply with the current divergence and vorticity estimates $\xi$ and $\zeta$, through a quadratic goodness-of-fit enforcement. The second part equips the divergence and the vorticity estimates with a robust first-order regularization favoring piecewise smooth configurations. Getting rid of the auxiliary scalar fields $\xi$ and $\zeta$ in (9) (by setting $\xi=\operatorname{div} \boldsymbol{w}$ and $\zeta=\operatorname{curl} \boldsymbol{w}$ ) would amount to the original secondorder div-curl regularization (8), if $\rho$ is the quadratic penalty function.

From a computational point of view, regularizing functional (9) only implies the numerical resolution of first-order PDE's. It is shown in the appendix that, at least for the $L_{2}$ norm, the regularization we propose is a smoothed version of the original second order div-curl regularization.

\subsection{Minimization Issue}

We now turn to the minimization issue of the whole energy function $H=$ $H_{1}+H_{2}$. Two main sets of variables have to be estimated. The first one is the motion field $\boldsymbol{w}$, and the second one consists in the two scalar fields $\xi$ and $\zeta$. The estimation is conducted alternatively by minimizing $H_{1}+H_{2}$ with respect to $\boldsymbol{w}, \xi$ and $\zeta$ respectively. For the motion field, considering the div and curl estimates $\xi$ and $\zeta$ as being fixed, the robust minimization with respect to $\boldsymbol{w}$ is solved with an iteratively reweighted least squares technique. This optimization is embedded in an efficient multi-parametric adaptive multigrid framework. In turn, $\boldsymbol{w}$ being fixed, the minimization of $H$ with respect to $\xi$ and $\zeta$ is in fact equivalent to the minimization of $\mathrm{H}_{2}$ and is again conducted using an iteratively 
reweighted least squares technique. More details of the minimization issues can be seen in 7.

Dense displacements fields obtained by minimizing the proposed energy functional, with its data-term based on the integrated continuity equation and its compound div-curl regularization, will be presented in section 5. Before we address in the two next sections the issue of analyzing resulting motion fields in terms of potential functions and singularities.

\section{Planar Vector Fields}

In this section, we present known analytic results on planar vector fields. We shall rely on them to develop an original method to extract singularities, streamfunctions and velocity-potentials, and parametric descriptions from the motion fields extracted with the method presented in previous section.

A planar vector field $\boldsymbol{w}$ is a $\mathbb{R}^{2}$-valued map defined on a bounded set $\Omega$ of $\mathbb{R}^{2}$. We note $\boldsymbol{w}(\boldsymbol{s}) \triangleq(u(\boldsymbol{s}), v(\boldsymbol{s}))$ and we assume that each component of the vector field is twice continuous and differentiable: $u$ and $v \in C^{2}(\Omega, \mathbb{R})$.

A vector field whose divergence is null everywhere is called solenoidal, and a vector field whose curl vanishes identically is called irrotational. A classical result, coming from the application of Green theorem shows that for irrotational fields there exists a scalar function $\phi$, called the velocity potential, such that $\boldsymbol{w}=\boldsymbol{\nabla} \phi$. Similarly, for solenoidal fields there exists a scalar function $\psi$ called the stream function such that $\boldsymbol{w}^{\perp}=\nabla \psi$, with $\boldsymbol{w}^{\perp}=(-v, u)^{T}$ is the orthogonal field of $\boldsymbol{w}$. The equipotential curves, $\{\psi(x, y)=c\}$, are the streamlines of the flow. For a flow both irrotational and solenoidal, it is interesting to note that level curves of $\phi$ and $\psi$ form an orthogonal network.

Irrotational and solenoidal fields play an important role in vector field analysis. As a matter of fact these two types of field can be combined to represent uniquely any arbitrary continuous vector field which vanishes at infinity. This is the Helmholtz representation of vector fields: $\boldsymbol{w}=\boldsymbol{w}_{\text {so }}+\boldsymbol{w}_{i r}$ (with $\boldsymbol{w}_{i r}=\boldsymbol{\nabla} \phi$ and $\boldsymbol{w}_{\text {so }}^{\perp}=\boldsymbol{\nabla} \psi$ ), where $\phi$ and $\psi$ are respectively the velocity potential of the irrotational component, and the stream function of the solenoidal part. When the null border condition at infinity can not be imposed, the representation is extended by the introduction of a third laminar component. A laminar field is a vector field that is both irrotational and solenoidal. The extended Helmholtz representation is then: $\boldsymbol{w}=\boldsymbol{w}_{\text {lam }}+\boldsymbol{w}_{\text {so }}+\boldsymbol{w}_{\text {ir }}$. In our applications, the laminar component accounts for a global transportation flow and for the effect of sources/sinks or vortices outside of the image plane. In the following we assume that this very smooth component is known. In practice, a sensible estimate of the laminar component can be obtained with standard motion estimation techniques under strong regularization. From now we will always refer to motion fields vanishing at infinity, and consequently to the original Helmholtz representation. 


\subsection{Irrotational and Solenoidal Field Separation}

Taking the divergence of $\boldsymbol{w}_{i r}$ and $\boldsymbol{w}_{s o}^{\perp}$ leads to $\boldsymbol{\nabla}^{2} \phi=\operatorname{div}(\boldsymbol{w})$ and $\boldsymbol{\nabla}^{2} \psi=$ $\operatorname{curl}(\boldsymbol{w})$. Both potential functions are therefore the solution of Poisson equations. Assuming that the curl and divergence vanish at infinity, one has to face a well known Dirichlet problem whose solution may be obtained through convolution with $2 D$ Green kernel. This direct solving is numerically tricky to implement since Green kernel lies on infinite support. Instead of that, using a spectral Fourier representation of the flow $\hat{\boldsymbol{w}}=(\mathcal{F}[u], \mathcal{F}[v]$ ) (such that $\mathcal{F}[f]=\hat{f}(\boldsymbol{k})=$ $\frac{1}{2 \pi} \iint f(\boldsymbol{s}) e^{-i<\boldsymbol{k}, \boldsymbol{s}>} d x d y$, with $\boldsymbol{k}=(\alpha, \beta)$ and $\left.\boldsymbol{s}=(x, y)\right)$ we have:

$$
\begin{aligned}
\mathcal{F}\left[\operatorname{curl}\left(\boldsymbol{w}_{i r}\right)\right] & =<\boldsymbol{k}^{\perp}, \hat{\boldsymbol{\omega}}_{i r}(\boldsymbol{k})>=0, \\
\mathcal{F}\left[\operatorname{div}\left(\boldsymbol{w}_{\text {so }}\right)\right] & =<\boldsymbol{k}, \hat{\boldsymbol{w}}_{\text {so }}(\boldsymbol{k})>=0,
\end{aligned}
$$

where $\langle\cdot, \cdot\rangle$ is the scalar product. Therefore, assuming the vector field $\boldsymbol{w}$ is known, the irrotational and the solenoidal components can be respectively obtained through:

$$
\hat{\boldsymbol{w}}_{i r}(\boldsymbol{k})=<\boldsymbol{k}, \hat{\boldsymbol{w}}(\boldsymbol{k})>\frac{\boldsymbol{k}}{\|\boldsymbol{k}\|^{2}}, \text { and } \hat{\boldsymbol{w}}_{s o}(\boldsymbol{k})=<\boldsymbol{k}^{\perp}, \hat{\boldsymbol{w}}(\boldsymbol{k})>\frac{\boldsymbol{k}^{\perp}}{\|\boldsymbol{k}\|^{2}},
$$

and the inverse Fourier transform.

\subsection{Potential Functions Estimation}

As we saw in the previous section, the knowledge of functions $\phi$ and $\psi$ might be very useful as it allows a complete description of the velocity field. In turn, if the velocity field and its irrotational and solenoidal components are known, they can be easily estimated (as $\boldsymbol{w}_{i r}=\boldsymbol{\nabla} \phi$ and $\boldsymbol{w}_{s o}^{\perp}=\boldsymbol{\nabla} \psi$ ). Noting that, if $g$ is a $C^{2}$ function, $g(x, y)=g(0,0)+\int_{\gamma} \nabla g(x, y) \cdot d \gamma$, where $\gamma$ is any path from $(0,0)$ to $(x, y)$. Averaging this relation over the two paths joining $(0,0)$ to $(x, y)$ along the sides of a rectangle, we get, taking $\phi(0,0)=\psi(0,0)=0$ :

$$
\left\{\begin{array}{l}
\phi(x, y)=\frac{1}{2}\left(\int_{0}^{x} u_{i r}(t, y) d t+\int_{0}^{y} v_{i r}(x, t) d t+\int_{0}^{x} u_{i r}(t, 0) d t+\int_{0}^{y} v_{i r}(0, t) d t\right), \text { and } \\
\psi(x, y)=\frac{1}{2}\left(\int_{0}^{y} u_{s o}(x, t) d t-\int_{0}^{x} v_{s o}(t, y) d t+\int_{0}^{y} u_{s o}(0, t) d t-\int_{0}^{x} v_{s o}(t, 0) d t\right) .
\end{array}\right.
$$

All terms of relation (12) may be numerically computed. They consist in integrations along the rows and the columns of the image.

\subsection{Extrema of the Potential Function}

It can be observed that characteristic points of the irrotational flow component (i.e., points $\boldsymbol{s}$ for which $\boldsymbol{w}_{i r}(\boldsymbol{s})=\boldsymbol{\nabla} \phi(\boldsymbol{s})=0$ ) corresponds to local extrema of the velocity potential $\phi$. Of course the same relation links extrema of the stream function and characteristic points of the solenoidal component. In addition, around 
a singular point $s$, the velocity distribution of a fluid flow can be accurately approximated (and characterized) by a so-called linear phase portrait [2. Within some neighborhood around $s$, one can fit a parametric velocity model of the form $\boldsymbol{w}=A s$ where $A$ is a $2 \times 2$ matrix. The qualitative characterization of the motion field in the neighborhood of this singular point $s$ relies on the structure of matrix $A$. Six typical motion configurations can be identified from its canonical Jordan form [211. A second-order approximation of the velocity potential and the stream function around a singular point, $\boldsymbol{w}_{i r}=\boldsymbol{\nabla} \phi(\boldsymbol{s}+\boldsymbol{\epsilon})=H_{\phi}(\boldsymbol{s}) \boldsymbol{\epsilon}+o(\boldsymbol{\epsilon})$ and $\boldsymbol{w}_{s o}^{\perp}=\nabla \psi(\boldsymbol{s}+\boldsymbol{\epsilon})=H_{\psi}(\boldsymbol{s}) \boldsymbol{\epsilon}+o(\boldsymbol{\epsilon})$, provides phase portraits $A_{\phi}=\left[\begin{array}{cc}\frac{\partial^{2} \phi}{\partial x^{2}} & \frac{\partial^{2} \phi}{\partial x \partial y} \\ \frac{\partial^{2} \phi}{\partial x \partial y} & \frac{\partial^{2} \phi}{\partial y^{2}}\end{array}\right]$ and $A_{\psi}=\left[\begin{array}{cc}\frac{\partial^{2} \psi}{\partial x \partial y} & \frac{\partial^{2} \psi}{\partial y^{2}} \\ -\frac{\partial^{2} \psi}{\partial x^{2}} & -\frac{\partial^{2} \phi}{\partial x \partial y}\end{array}\right]$. Matrix $A_{\phi}$ is symmetric (it has real eigenvalues) and positive definite or negative definite around local extrema (the eigenvalues have all the same sign). The corresponding singular point is therefore a node or a star node (cf. [2]) which depicts well the behavior of sources or sinks. Concerning the solenoidal field, trace of $A_{\psi}$ is null: the local extremum then corresponds to the singular point at the center of a rotating motion. This is the characterization of a vortex.

\subsection{Rankine Model of Flows}

One of the simplest models of velocity field for fluid flows at singularities is provided by the Rankine model of vortex [6]. It consists in approximating the velocity field as a vector field of constant curl inside a disk and null curl beyond this circular domain. The complex function $f(z)=u(x, y)+i v(x, y)$ associated to this velocity field reads:

$$
f_{\imath}(z) \triangleq\left\{\begin{array}{lll}
g_{\imath}(z)=-\frac{i \beta_{\imath}\left(z-z_{\imath}\right)}{\left|z-z_{\imath}\right|^{2}} & \text { if } & \left|z-z_{\imath}\right| \geq r_{\imath} \\
h_{\imath}(z)=-\frac{i \beta_{\imath}\left(z-z_{\imath}\right)}{r_{\imath}^{2}} & \text { if } & \left|z-z_{\imath}\right|<r_{\imath}
\end{array}\right.
$$

where $r_{\imath}$ is the singularity radius, $z_{\imath} \triangleq x_{\imath}+i y_{\imath}$ denotes the complex vortex location, and $\beta_{\imath}$ its strength. Based on a similar model, the velocity field associated to a source/sink in the plane can modeled as:

$$
f_{\jmath}(z) \triangleq\left\{\begin{array}{lll}
g_{\jmath}(z)=\frac{\alpha_{\jmath}\left(z-z_{\jmath}\right)}{\left|z-z_{\jmath}\right|^{2}} & \text { if } & \left|z-z_{\jmath}\right| \geq r_{\jmath} \\
h_{\jmath}(z)=\frac{\alpha_{\jmath}\left(z-z_{\jmath}\right)}{r_{\jmath}^{2}} & \text { if } & \left|z-z_{\jmath}\right|<r_{\jmath}
\end{array}\right.
$$

where $\alpha_{\jmath}$ denotes the sink/source's strength. If $\alpha_{\jmath}>0$, this constitutes a source model, whereas if $\alpha_{\jmath}<0$ we are in presence of a sink. It is easy to verify that $i$ ) function $f_{\imath}$ in (13) defines a solenoidal field, and function $f_{\jmath}$ in (14) defines an 
irrotational field; $i i)$ functions $g_{\imath}$ is such that $\operatorname{curl} g_{\imath}=\operatorname{div} g_{\jmath}=0$ and function $h_{\imath}$ is such that $\operatorname{curl} h_{\imath}=\frac{2 \beta_{\imath}}{r_{\imath}^{2}}, \operatorname{div} h_{\jmath}=\frac{2 \alpha_{\jmath}}{r_{\jmath}^{2}}$.

These two types of fields can be composed to model a fluid flow with $P$ vortices and $N$ sources/sinks within $f(z)=\sum_{\imath=1}^{P} f_{\imath}(z)+\sum_{\jmath=1}^{N} f_{\jmath}(z)$.

\section{Motion-Based Segmentation Based on Rankine Model Around Singularities}

A global description of the flow based on the Rankine model allows to define a main characteristics of the flow in terms of interacting singularities and influence circular domains. Let us show how all the ingredients of such a motion-based segmentation may be identify from a dense motion field.

\subsection{Localisation of Singular Points}

Given a motion field $\boldsymbol{w}$ obtained by means of the technique introduced in section 2, the spectral technique described in section 3.1 enables to recover the associated stream-function $\phi$ and velocity potential $\psi$. We saw also that the knowledge of both potential functions gives a practical way to identify all the vortices and sinks/sources of the flow by extracting their respective extrema. In practice, these extrema are obtained through a simple morphological processing of the potential functions. Both are derived from dense motion field Helmholtz components $\boldsymbol{w}_{i r}=\boldsymbol{\nabla} \phi$ and $\boldsymbol{w}_{s o}^{\perp}=\nabla \psi$, using (12). Note that the laminar part of the motion field is assumed to be well approximated by the smooth motion field estimate obtained at the coarsest level of our multigrid setting.

\subsection{Extraction of Rankine Parameters}

Assuming that all singularities of the flow are known, a complete parametric representation of the flow as a superposition of individual rotational and divergent Rankine models can be sought. To this end, we need to estimate the strength and the circular linearity domain associated to each singular point.

Assuming that the solenoidal and irrotational components of the flow differ from the two corresponding components of the compound Rankine model by a white Gaussian noise of variance $\sigma^{2}$, we get:

$$
f_{s o}(z)=\sum_{\imath=1}^{P}\left(f_{\imath}(z)+a(z)+i b(z)\right) \quad \text { and } \quad f_{i r}(z)=\sum_{\jmath=1}^{N}\left(f_{\jmath}(z)+a(z)+i b(z)\right)
$$

with $a(z)$ and $b(z) \sim \mathcal{N}\left(0, \sigma^{2}\right)$. Function $f_{s o} \triangleq u_{s o}+i v_{s o}$ (resp. $f_{i r} \triangleq u_{i r}+$ $i v_{i r}$ ) is the complex representation of $\boldsymbol{w}_{s o}$ (resp. of $\boldsymbol{w}_{i r}$ ), and $P$ and $N$ denote respectively the number of vortices and sources/sinks of the flow. 
A maximum likelihood estimation of the Rankine model parameters leads to maximize with respect to the unknown parameters vector $\Theta \triangleq\left(r_{\imath}, \beta_{\imath}\right)_{\imath=1}^{P} \times$ $\left(r_{\jmath}, \alpha_{\jmath}\right)_{\jmath=1}^{N}$ the following log-likelihood defined on the whole image domain $\Omega$ :

$$
\mathcal{L}(\Theta)=\underbrace{\iint_{\Omega}\left|f_{\text {ir }}(z)-\sum_{\imath} f_{\imath}(z)\right|^{2} d z}_{\mathcal{L}_{\text {so }}}+\underbrace{\iint_{\Omega}\left|f_{\text {so }}(z)-\sum_{\jmath} f_{\jmath}(z)\right|^{2} d z}_{\mathcal{L}_{\text {ir }}} .
$$

With the assumption that two circular linearity domains of the same nature do not intersect each other, the two parts of this expression can be expressed as:

$$
\mathcal{L}_{\text {so }}(\Theta)=\sum_{\imath=1}^{P} \iint_{\mathcal{D}_{\imath}}\left|f_{\text {so }}(z)-h_{\imath}\left(r_{\imath}, z\right)-\sum_{k \neq \imath} g_{k}(z)\right|^{2} d z+\iint_{\overline{\mathcal{D}}}\left|f_{\text {so }}(z)-\sum_{\imath=1}^{P} g_{p}(z)\right|^{2} d z
$$

and similarly for $\mathcal{L}_{i r}$, where $\mathcal{D}_{\imath}$ denotes the disk associated to the $\imath$ th vortex singularity and $\overline{\mathcal{D}}_{\imath} \triangleq \Omega-\bigcup_{\imath=1}^{P} \mathcal{D}_{\imath}$. It is important to remark that the nonoverlapping assumption only apply to domains associated to singularities of the same type. Likelihood (16) is still valid for a vortex and a source combined in a swirl.

Expanding expression (16) in the solenoidal case (the same computations may be carried out in the irrotational case) one gets:

$$
\begin{aligned}
\mathcal{L}_{s o}(\Theta) & =\sum_{\imath} \iint_{\mathcal{D}_{\imath}}\left\|\boldsymbol{w}_{s o}(s)+\frac{\left(s-s_{\imath}\right)^{\perp}}{r_{i}^{2}} \beta_{\imath}+\sum_{k \neq \imath} \frac{\left(s-s_{k}\right)^{\perp}}{\left\|s-s_{k}\right\|^{2}} \beta_{k}\right\|^{2} d s \\
& +\iint_{\overline{\mathcal{D}}}\left\|\boldsymbol{w}_{s o}(s)+\sum_{k} \frac{\left(s-s_{k}\right)^{\perp}}{\left\|s-s_{k}\right\|^{2}} \beta_{k}\right\|^{2} d s .
\end{aligned}
$$

A maximizer of this likelihood is given by solving $\boldsymbol{\nabla} \mathcal{L}_{s o}=0$, where $\boldsymbol{\nabla}=$ $\left(\frac{\partial}{\partial r_{1}}, \ldots, \frac{\partial}{\partial r_{N}}, \frac{\partial}{\partial \beta_{1}}, \ldots, \frac{\partial}{\partial \beta_{N}}\right)^{T}$. The cancellation of the partial derivative w.r.t. the $r_{\imath}$ 's leads to (remarking with some efforts that $\frac{\partial}{\partial R}\left(\iint_{\mathcal{D}} f+\iint_{\overline{\mathcal{D}}} g\right)=\iint_{\mathcal{D}} \frac{\partial f(R)}{\partial R}+$

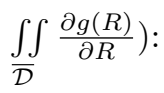

$$
\frac{\pi}{2} r_{\imath}^{2}=-\frac{1}{\beta_{\imath}} \iint_{\mathcal{D}_{\imath}}\left[\left(\boldsymbol{w}_{s o}(s)+\sum_{k \neq \imath} \frac{\left(s-s_{k}\right)^{\perp}}{\left\|s-s_{k}\right\|^{2}} \beta_{k}\right) \cdot\left(s-s_{\imath}\right)^{\perp}\right] d s .
$$


Concerning the $\beta_{\imath}$ 's we get:

$$
\begin{aligned}
& \beta_{\imath}=\frac{B+C}{A}, \text { with } \\
& A=\iint_{\Omega-\mathcal{D}_{\imath}} \frac{1}{\left\|s-s_{\imath}\right\|^{2}} d s, B=\iint_{\overline{\mathcal{D}}}\left[\boldsymbol{w}_{s o}(s)+\sum_{k \neq \imath} \frac{\left(s-s_{k}\right)^{\perp}}{\left\|s-s_{k}\right\|^{2}} \beta_{k}\right] \cdot \frac{\left(s-s_{\imath}\right)^{\perp}}{\left\|s-s_{\imath}\right\|^{2}} d s, \\
& C=\sum_{k \neq \imath} \iint_{\mathcal{D}_{k}}\left[\boldsymbol{w}_{s o}(s)+\frac{\left(s-s_{k}\right)^{\perp}}{r_{k}^{2}} \beta_{k}+\sum_{p \notin\{k, \imath\}} \frac{\left(s-s_{p}\right)^{\perp}}{\left\|s-s_{p}\right\|^{2}} \beta_{p}\right] \cdot \frac{\left(s-s_{\imath}\right)^{\perp}}{\left\|s-s_{\imath}\right\|^{2}} .
\end{aligned}
$$

Equations 18 and 19 are solved alternatively. For fixed radius, 19 defines a linear system of equations w.r.t. the $\beta_{\imath}$ 's. In turn, the strength parameters $\beta_{\imath}$ 's being fixed, the independent non-linear equations (18) are solved with a kind of fixed point method: the integral is computed using the previous estimate of radius $r_{\imath}$ (the initial radius is fixed to a small value (3 pixels in practice). The resolution of both systems is iterated until convergence (see 8] for details explanations). Let us note that an additional constraint which guaranty nonoverlapping domains must be included to ensure an admissible solution.

\subsection{Elimination of Noisy Singularities}

In order to keep only the most significant singularities to describe the motion field, we consider the Bhattacharyya distance between two multidimensional Gaussian laws [3]:

$$
d_{B}\left[\mathcal{N}_{1}\left(\boldsymbol{\mu}_{\mathbf{1}}, \Sigma_{1}\right), \mathcal{N}_{2}\left(\boldsymbol{\mu}_{\mathbf{2}}, \Sigma_{2}\right)\right]=\frac{1}{4}(\boldsymbol{\mu})^{T}\left(\Sigma_{1}+\Sigma_{2}\right)^{-1}(\boldsymbol{\mu})+\frac{1}{2} \ln \left(\frac{\operatorname{det}\left(\Sigma_{2}+\Sigma_{1}\right)}{2 \sqrt{\operatorname{det}\left(\Sigma_{1} \Sigma_{2}\right)}}\right)
$$

with $\boldsymbol{\mu}=\boldsymbol{\mu}_{\mathbf{2}}-\boldsymbol{\mu}_{\mathbf{1}}$. For each component (i.e., the irrotational one or the solenoidal one) we compute this distance for the two Gaussian distributions corresponding to the error between the considered Rankine model and the dense motion field for two consecutive numbers of singularities. For example for the solenoidal component we compute: $d_{B}\left[\mathcal{N}_{1}\left(\boldsymbol{\mu}^{n}, \Sigma^{n}\right), \mathcal{N}_{2}\left(\boldsymbol{\mu}^{n+1}, \Sigma^{n+1}\right)\right]$ where, $\boldsymbol{\mu}^{k}$ and $\Sigma^{k}$ are the mean and the variance of the difference field $\left(\boldsymbol{w}_{s o}-\boldsymbol{w}_{\Theta_{s o}}^{k}\right)$. Parametric field $\boldsymbol{w}_{\Theta_{s o}}^{k}$ correspond to a maximum likelihood estimate of Rankine model with $k$ vortices. Starting with no singularities, we increase the number of singularities by considering the largest local maxima of its corresponding squared potential function. When the Bhattacharyya distance between two consecutive models is small enough (i.e., when the introduction of a new singularity does not bring additional information) the process is stopped.

\section{Experimental Results}

In this section we present some experimental results. Presented examples correspond to the motion between two consecutive images of the infra-red channel of 
Meteosat, shot the $21^{\text {st }}$ of January 1998 (Figs. 1 1a-b), and between two consecutive images of the water-vapor channel of Meteosat, shot the $4^{\text {th }}$ of August 1995 (Figs. 1k-d). Both examples images exhibit a large through of low pressure. In addition, the two first images exhibit a set of moving cloud structures (top-right part of the image) and the two others an exploding convective cell. Corresponding estimated vector fields with their laminar component removed are visible in Figs. 2a and 2b. These motion fields seem visually plausible: both the main structures of the motion (counter-clockwise spiral of depressions, downward motion of cold clouds for the infrared image, the convective cell for the water vapor image) are captured.

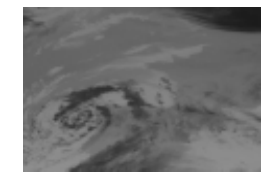

(a)

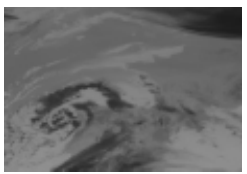

(b)

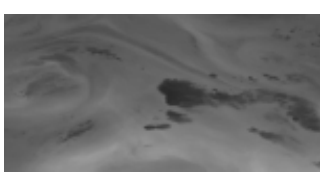

(c)

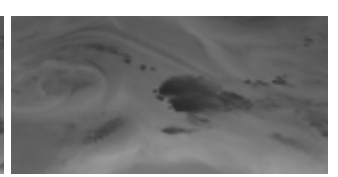

(d)

Fig. 1. Two consecutive images of each sequence treated: $(a, b)$ Infrared images; $(c, d)$ Water vapor images

The solenoidal and the irrotational components of these velocity fields are shown in the second column of Fig. 2. In the third column, we present for the first example the squared potential functions from which we extract the singularities by localizing their extrema. For the second example, we present the stream lines and the level curves of the velocity potential. The estimated singularity domains are superimposed to their corresponding motion fields. The parametric fields associated to estimated compound Rankine models are shown in Figs. [2d and [2].

This method captures the main visible structures of the flow (seven vortices and four sources, for the first example, four vortices and one source for the second example). The associated parametric motion fields constitute fair "summaries" of the structure of the flows.

\subsection{Comparison with Winding Number Technique}

A popular method to extract singular points is based on the use of Poincaré indices also called winding numbers. The winding number of a closed curve in a vector field amounts to the number of turns, $\frac{1}{2 \pi} \int d\left(\tan ^{-1} u / v\right)$, that the field undergoes along the curve. Its value is +1 if the considered Jordan curve surrounds a vortex/sink/source. In practice, due to the image discretization, a small blob (whose size depends on the size of used curve) of +1 index pixels is obtained in the neighborhood of a singular point.

This method as the advantage to be fast. Nevertheless, it remains based on a local criterion which is not robust to noise. Furthermore, only blobs containing a 


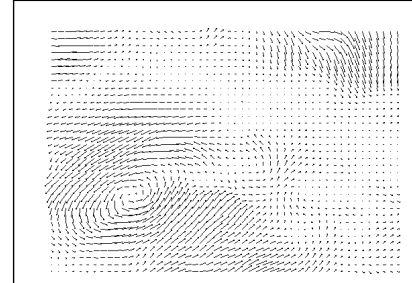

(a)

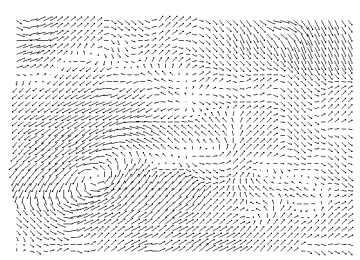

(d)

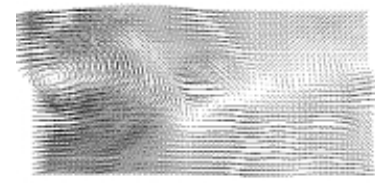

(g)

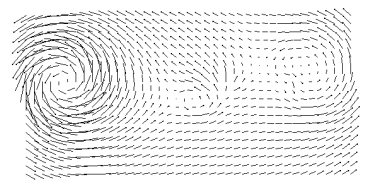

(j)

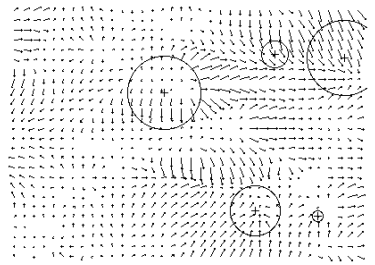

(b)

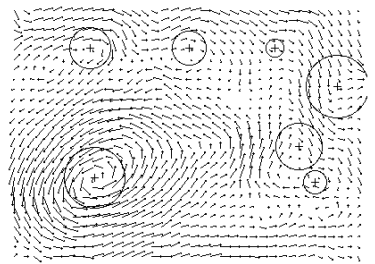

(e)

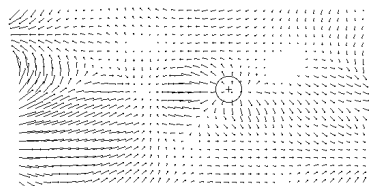

(h)

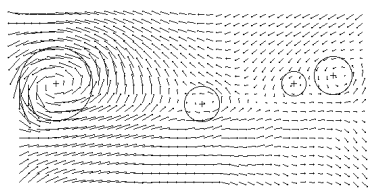

(k)

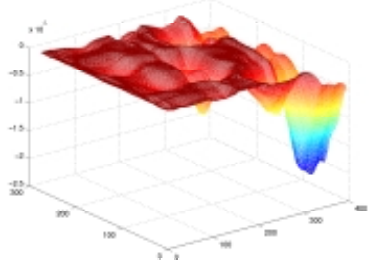

(c)

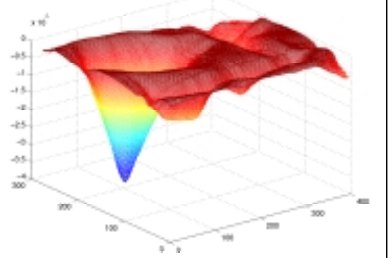

(f)

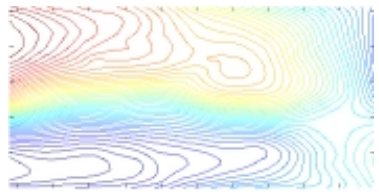

(i)

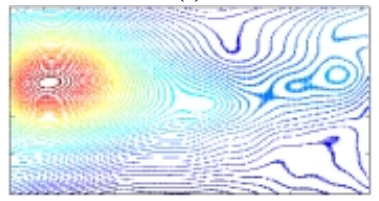

(I)

Fig. 2. (a-f) results for the IR images presented in Fig. 19 and ( $g-I)$ results for the WV images presented in Fig. 1 $1 \mathrm{~b}-(\mathrm{a}, \mathrm{g})$ : dense motion fields obtained with the proposed technique; $(d, j)$ : corresponding parametric Rankine flows; $(b, h)$ : estimated sources/sinks superimposed to the irrotational part of the flow; $(\mathrm{e}, \mathrm{k})$ : estimated vortices superimposed to the solenoidal part of the flow; $(c, f)$ : squared velocity potential and squared stream function; $(\mathrm{i}, \mathrm{I})$ : stream lines $(\psi=$ constant) and level curves of the velocity potential $(\phi=$ constant $)$.

potential singular point may be detected with such technique. The exact location of the singularity has then to be extracted from such blobs with other adhoc techniques.

In order to illustrate the difference between such an approach and the one we propose, we present in Fig. 3, the different blobs detected via winding indices, from to dense motion fields estimated with the dedicated approach. Figure 3 a corresponds to the singularities detected for the infrared sequence, whereas Fig. 36 corresponds to the ones obtained for the water-vapor example. 
We can note that the correct singular points are also detected at least within the few pixel location accuracy associated to each blob. Nevertheless, the results are cluttered by a large number of false positives due to the sensitivity of the technique. These spurious points have then to be removed with some post-processing treatments.

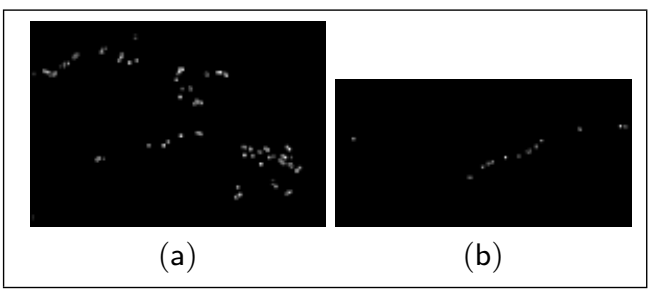

Fig. 3. Blobs of singularities estimated with winding number technique; (a) blobs corresponding to the infrared example and (b) blobs corresponding to the water vapor example .

\section{Conclusion}

In this paper, we have presented a complete method to analyze fluid flows from image sequences. We first presented a dedicated technique to estimate dense motion fields. This method modifies generic energy-based robust techniques through a new data term based on the continuity equation, and an original smoothness prior which preserves high concentrations of divergence and curl. We then have proposed an original technique to detect singular points and their associated domain of linearity from a dense motion field. This technique is based on the numerical decomposition of the motion field in terms of its irrotational and solenoidal components. From these components, we extract by integration the associated stream function and the velocity potential, whose local extrema provide the location of vortices and sinks/sources. The strength and linearity domain associated to each of these detected singular points are then obtained from a maximum likelihood estimation of a parametric Rankine model.

The whole approach has been demonstrated on two real meteorological examples. In [7, we shows that the fluid motion estimator provide better results than generic motion estimators based on brightness constancy assumption and a firstorder smoothness. The singularities extraction method described here allows to extract the main structures of a motion field. Compared to an usual winding number technique, our approach is more robust to various sources of noise. As a by product, our approach provides a simple way to extract streamlines, velocity potential, solenoidal or irrotational components, which are central to many studies of fluids. As a final remark let us outline that the method described here requires no tuning of parameters. 


\section{References}

1. A. Amini. A scalar function formulation for optical flow. In Proc. Europ. Conf. Computer Vision, pages 125-131, 1994.

2. V.I. Arnold. Ordinary differential equations. MIT Press, 1990.

3. M. Basseville. Distance measure for signal processing and pattern recognition. Signal Processing, (18):349-369, 1989.

4. D. Béréziat, I. Herlin, and L. Younes. A generalized optical flow constraint and its physical interpretation. In Proc. Conf. Comp. Vision Pattern Rec., volume 2, pages 487-492, Hilton Head Island, South Carolina, USA, 2000.

5. M. Black and P. Anandan. The robust estimation of multiple motions: Parametric and piecewise-smooth flow fields. Computer Vision and Image Understanding, 63(1):75-104, 1996.

6. A. J. Chorin. Vorticity and turbulence. Applied Math. Sciences 103, Springer Verlag.

7. T. Corpetti, E. Mémin, and P. Pérez. Dense estimation of fluide flows. IEEE Trans on Pattern Analysis and Machine Intelligence, 24(3), March 2002.

8. T. Corpetti, E. Mémin, and P. Pérez. Extraction of singular points from dense motion fields: an analytic approach. Journal of Math. Imag. and Vision, 2002. Accepted under minor revisions.

9. S. Das Peddada and R. McDevitt. Least average residual algorithm (LARA) for tracking the motion of artic sea ice. IEEE trans. on Geosciences and Remote sensing, 34(4):915-926, 1996.

10. J.M. Fitzpatrick. The existence of geometrical density-image transformations corresponding to object motion. Comput. Vision, Graphics, Image Proc., 44(2):155$174,1988$.

11. R.M. Ford, R. Strickland, and B. Thomas. Image models for 2-d flow visualization and compression. Graph. Mod. Image Proc., 56(1):75-93, 1994.

12. S. Gupta and J. Prince. Stochastic models for div-curl optical flow methods. Signal Proc. Letters, 3(2):32-34, 1996.

13. B. Horn and B. Schunck. Determining optical flow. Artificial Intelligence, 17:185203, 1981.

14. P. Kornprobst, R. Deriche, and G. Aubert. Image sequence analysis via partial differential equations. Journal of Mathematical Imaging and Vision, 11(1):5-26, September 1999.

15. R. Larsen, K. Conradsen, and B.K. Ersboll. Estimation of dense image flow fields in fluids. IEEE trans. on Geoscience and Remote sensing, 36(1):256-264, 1998.

16. E. Mémin and P. Pérez. Dense estimation and object-based segmentation of the optical flow with robust techniques. IEEE Trans. Image Processing, 7(5):703-719, 1998.

17. E. Mémin and P. Pérez. Hierarchical estimation and segmentation of dense motion fields. Int. J. Computer Vision, 46(2):129-155, February 2002.

18. A. Nomura, H. Miike, and K. Koga. Field theory approach for determining optical flow. Pattern Recognition Letters, 12(3):183-190, 1991.

19. A. Ottenbacher, M. Tomasini, K. Holmund, and J. Schmetz. Low-level cloud motion winds from Meteosat high-resolution visible imagery. Weather and Forecasting, 12(1):175-184, 1997.

20. B.G. Schunk. The motion constraint equation for optical flow. In Proc. Int. Conf. Pattern Recognition, pages 20-22, Montreal, 1984. 
21. S.M. Song and R.M. Leahy. Computation of 3D velocity fields from 3D cine and CT images of human heart. IEEE trans. on medical imaging, 10(3):295-306, 1991.

22. D. Suter. Motion estimation and vector splines. In Proc. Conf. Comp. Vision Pattern Rec., pages 939-942, Seattle, USA, June 1994.

23. R. Wildes, M. Amabile, A.M. Lanzillotto, and T.S. Leu. Physically based fluid flow recovery from image sequences. In Proc. Conf. Comp. Vision Pattern Rec., pages 969-975, 1997.

24. L. Zhou, C. Kambhamettu, and D. Goldgof. Fluid structure and motion analysis from multi-spectrum 2D cloud images sequences. In Proc. Conf. Comp. Vision Pattern Rec., volume 2, pages 744-751, Hilton Head Island, South Carolina, USA, 2000.

\section{Appendix}

In this appendix, we demonstrate (for the $L_{2}$ norm) that the regularization we propose in (9) corresponds to a smoothed form of the initial second-order regularization (8). To show that, let us consider only the div part of both functionals (the same deviations can be obtained for the curl part). Because of the Parseval theorem, the Fourrier transform of the first term of (8) verifies:

$$
\mathcal{F}\left(\int_{\Omega}\|\boldsymbol{\nabla} \operatorname{div} \boldsymbol{w}\|^{2} d s\right)=\int|\mathcal{F}(\boldsymbol{\nabla} \operatorname{div} \boldsymbol{w})|^{2} d \boldsymbol{k}=\int\|\boldsymbol{k}\|^{2}|\boldsymbol{k} \cdot \hat{\boldsymbol{w}}(\boldsymbol{k})|^{2} d \boldsymbol{k}
$$

Similarly, the Fourier transform of the corresponding term in (9) verifies (with $\mu=\frac{1}{\lambda}$ ):

$$
\mathcal{F}\left(\int_{\Omega} \mu^{2}(\operatorname{div} \boldsymbol{w}-\xi)^{2}+\|\nabla \xi\|^{2} d s\right)=\int\left(\mu^{2}|\boldsymbol{k} \cdot \hat{\boldsymbol{w}}(\boldsymbol{k})-\hat{\xi}|^{2}+\|\boldsymbol{k}\|^{2}|\hat{\xi}|^{2}\right) d \boldsymbol{k} .
$$

For a fixed $\boldsymbol{w}$, a minimizer of $(22)$ is given by the resolution of the EulerLagrange equation $\left(\mu^{2}(\operatorname{div} \boldsymbol{w}-\xi)+\Delta \xi=0\right)$. In the Fourrier domain, the minimizer is:

$$
\hat{\xi}_{\text {opt }}=\frac{\mu^{2}|\boldsymbol{k} \cdot \hat{\boldsymbol{w}}(\boldsymbol{k})|}{\|\boldsymbol{k}\|^{2}+\mu^{2}} .
$$

Introducing $\hat{\xi}_{\text {opt }}$ in (22), after few manipulations leads to:

$$
\begin{aligned}
\mathcal{F}\left(\int_{\Omega} \mu^{2}\left(\operatorname{div} \boldsymbol{w}-\xi_{\text {opt }}\right)^{2}+\left\|\boldsymbol{\nabla} \xi_{\text {opt }}\right\|^{2} d \boldsymbol{s}\right) & =\int \frac{\mu^{2}\|\boldsymbol{k}\|^{2}}{\|\boldsymbol{k}\|^{2}+\mu^{2}}|\boldsymbol{k} \cdot \hat{\boldsymbol{w}}(\boldsymbol{k})|^{2} d \boldsymbol{k} \\
& =\int \hat{g}(\boldsymbol{k})|\mathcal{F}(\|\nabla \operatorname{div} \boldsymbol{w}\|)|^{2} d \boldsymbol{k}
\end{aligned}
$$

with $\hat{g}(\boldsymbol{k})=\frac{\mu^{2}}{\mu^{2}+\|\boldsymbol{k}\|^{2}}$. With $\mu=\frac{1}{\lambda}$ (in that case, the regularization is the same than (9)), one get $\hat{g}(\boldsymbol{k})=\frac{1}{1+\lambda^{2}\|\boldsymbol{k}\|^{2}}$. For low frequencies, $\hat{g}(\boldsymbol{k}) \rightarrow 1$ and the regularization functions are the same. For high frequencies, $\hat{g}$ is a smoothing function. Nevertheless, as we assume that $\boldsymbol{w}$ is $C^{2}$, we have $\lim _{\|\boldsymbol{k}\| \rightarrow+\infty} \hat{\boldsymbol{w}} \cdot\|\boldsymbol{k}\|^{2}=$ 0 (i.e. $\hat{\boldsymbol{w}}$ tends to zero faster than $\frac{1}{\|\boldsymbol{k}\|^{2}}$ when $\boldsymbol{k}$ tends to $+\infty$ ). 